\title{
Lattice Registry and Evidence for Surface Reconstructions of Metal Films on Suspended 2D Membranes Following Annealing
}

\author{
Todd H. Brintlinger ${ }^{1 *}$, Jose Fonseca Vega ${ }^{2}$, James Clifford Culbertson ${ }^{2}$, Maxim Zalalutdinov ${ }^{3}$, \\ Rhonda M. Stroud ${ }^{1}$, and Jeremy T. Robinson ${ }^{2}$ \\ 1. Materials Sci. \& Tech. Division, U.S. Naval Research Laboratory, Washington, DC, USA 20375 \\ 2. Electronics Sci. \& Tech. Division, U.S. Naval Research Laboratory, Washington, DC, USA 20375 \\ 3. Acoustics Division, U.S. Naval Research Laboratory, Washington, DC, USA 20375 \\ * Corresponding author: todd.brintlinger@nrl.navy.mil
}

Since their isolation as atomically thin materials, the interaction of 2D crystals (2DCs) with the substrates with which they are in contact has been an enduring area of research. With insulating support substrates, trapped charge can radically alter both the electronic [1] and optical behavior [2] of the 2DC. While typically not employed as a substrate per se, metals can host 2DCs during growth, metals are ubiquitous as contact/electrode layers, and nanostructured metals are employed in interdisciplinary fields such as plasmonic-2DC coupling. The use of metals is also a means by which the underlying, or overlying, 2D materials can be manipulated in different environments, e.g. deployed as a sacrificial substrate, gold has been used in the production of large area $\left(\sim 1 \mathrm{~cm}^{2}\right)$ regions of 2D materials [3]. While most research has focused on how the substrate influences 2DC properties, we find that 2DCs measurably influence their metal hosts $[4,5]$, despite a drastic disparity in thickness.

In this effort, we examine the morphological influence that $\mathrm{MoS}_{2}$ monolayers have on relatively thick $(25 \mathrm{~nm})$ gold thin films during annealing. To form the cleanest interfaces with the highest crystalline quality, we exfoliate $\mathrm{MoS}_{2}$ onto Au thin films which have been evaporated onto polyvinyl alcohol (PVA) films which have been spun onto silicon dioxide chips, remove the sacrificial PVA film, and transfer the remaining $\mathrm{MoS}_{2} / \mathrm{Au}$ stack for annealing. Ex-situ annealing experiments of $\mathrm{MoS}_{2} / \mathrm{Au}$ on $\mathrm{SiO}_{2}$ substrates shows that the Au films can become textured (oriented), as revealed through electron beam scattering diffraction, and that the Au film can locally dewet beneath the $\mathrm{MoS}_{2}$ layer to form a porous metal layer with suspended $\mathrm{MoS}_{2}$ membranes. To further investigate this behavior, we imaged these films using aberration-corrected scanning transmission electron microscopy (Nion UltraSTEM-200X) after both exsitu and in-situ annealing.

For ex-situ annealing, $\mathrm{Au}_{25 \mathrm{~nm}} / \mathrm{MoS}_{2}$ films were transferred to $\mathrm{SiC}$ membranes covered with holey carbon (Protochips E-FHBC chips), annealed in a tube furnace at $300^{\circ} \mathrm{C}$ in a reducing environment, then baked again at $140^{\circ} \mathrm{C}$ for 14 hours in vacuum, and loaded into the STEM column. For in-situ annealing, samples were transferred onto similar substrates as for ex situ experiments, loaded into electrical biasing cartridges, baked for 14 hours at $140^{\circ} \mathrm{C}$, inserted into the microscope, and then we perform imaging as temperature is increased from $150^{\circ} \mathrm{C}$ to $400^{\circ} \mathrm{C}$. For all imaging, the microscope is operated at $60 \mathrm{kV}$ with a $\sim 100 \mathrm{pA}$ beam current using high- and medium-angle annular dark field detectors (HAADF and MAADF), as well as bright-field (BF) detector. After ex-situ annealing, we find a consistent lattice registry between the $\mathrm{MoS}_{2}$ and $\mathrm{Au}$ at a dewetted metal pore site, seen in Fig. 1. Here, we can show that not only is the gold film ordering, but it is doing so in registry with the $\mathrm{MoS}_{2}$ layer underneath. Furthermore, in Fig. 2, we note in the BF image an unexpected, regular striped pattern in the Au film that resembles the well-known $\mathrm{Au}$ 'herringbone' reconstruction (indicated by arrows) seen in scanning tunneling microscopy studies [6, 7]. Evidence for an otherwise strictly surface phenomena is remarkable for a thick gold film, imaged in 
transmission mode, and the origin of this contrast is yet to be definitively identified. Thus, we have multiple instances where an atomically thin 2DC can affect a much thicker, gold thin film.

\section{References:}

[1] W Bao et al., Appl Phys Lett 102 (2013), p. 042104.

[2] M Buscema et al., Nano Research 7 (2014), p. 561.

[3] MA Islam et al., Nano Lett 17 (2017), p. 6157.

[4] P Cao et al., Advanced Materials 29 (2017), p. 1701536.

[5] Y Liu et al., Nature 557 (2018), p. 696.

[6] C Wöll et al., Physical Review B 39 (1989), p. 7988.

[7] JV Barth et al., Physical Review B 42 (1990), p. 9307.
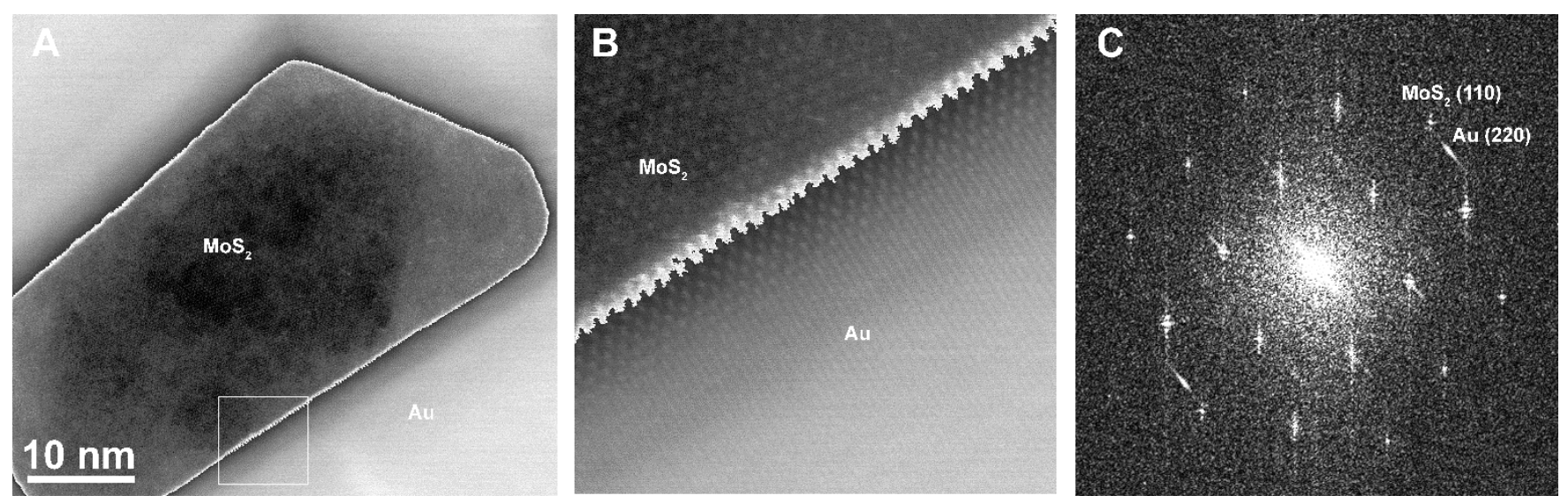

Figure 1. Aberration-corrected STEM of $\mathrm{MoS}_{2}$ transferred onto gold thin films and annealed at $300^{\circ} \mathrm{C}$. (A) HAADF micrograph of monolayer $\mathrm{MoS}_{2}$ surrounded by a $25 \mathrm{~nm}$ thick gold film, with histogram equalization applied to each region separately. (B) A higher-magnification view of the indicated box in (A) showing the interface between $\mathrm{MoS}_{2}$ and Au. (C) FFT of region shows registry of Au (220) and $\mathrm{MoS}_{2}$ (110) lattice.

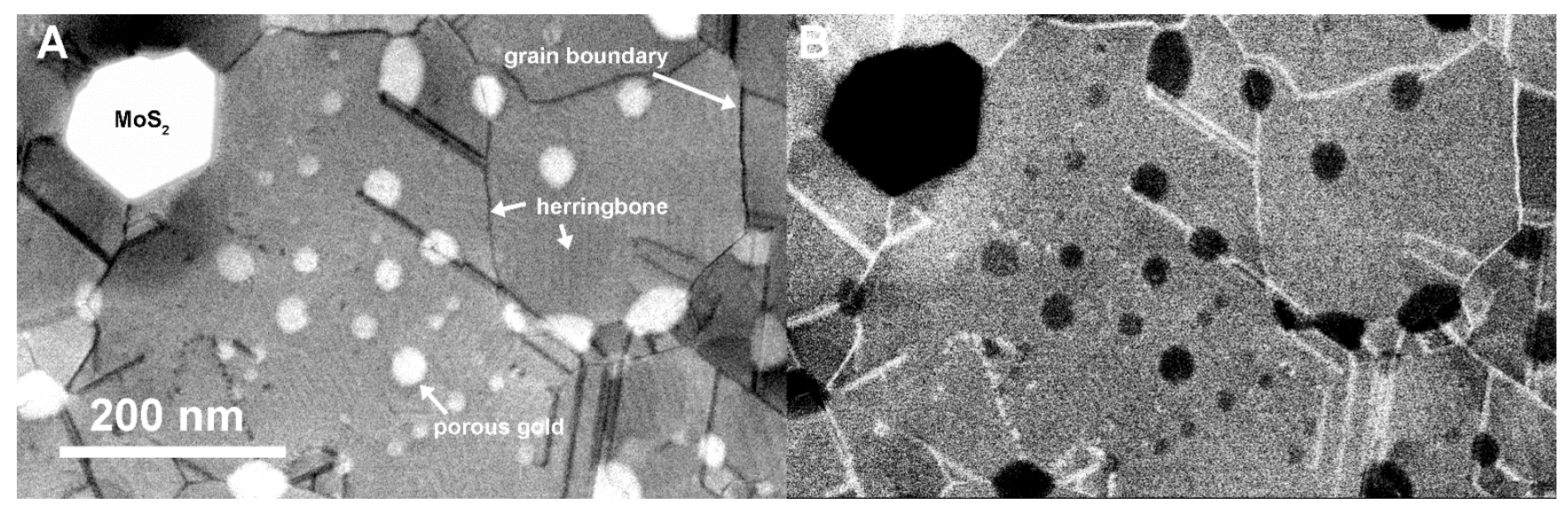

Figure 2. Low magnification STEM images of gold film with a monolayer $\mathrm{MoS}_{2}$ 'pore'. (A) BF STEM shows thinner regions of gold appearing as bright circles and grain boundaries appear dark. A small intensity variation (labeled 'herringbone') strongly resembles the surface reconstruction of Au (111) seen with scanning tunneling microscopy. (B) HAADF STEM reveals inverted contrast from BF STEM for $\mathrm{MoS}_{2}$ pore, grain boundaries, and thinner gold regions. 